[CONTRIBUTION FROM THE MELION INSTITUTE OF INDUSTRIAL RESEARCH OF THE UNIVERSITY OF PITTSBURGH. I

\title{
THE EFFECT OF SURFACE OXIDATION ON SOME METALLIC CATALYSTS. ${ }^{1}$
}

BY FRANK F. RUPERT. ${ }^{2}$

Received December 27, 1919.

A paper by Wigand entitled "The Increase of the Catalytic Action of Platinum on the Combination of Detonating Gas by Galvanic Polarization," suggested experiments leading to a further development of Wigand's theme. A repetition of his experiments with a simple form of

IThis paper describes part of an investigation made by the author in $19.3^{-14}$ on the general subject of the relation of electrical potential to catalysis. The study of a number of phases of the general problem gave entirely negative results in practice, and full consideration of the theoretical bearings of the problem afforded no justificam tion for its underlying premises. It was learned, however, that there are a few cases in which electrical potential and catalysis are indirectly related, although the connection may be accidental, and one of these cases, which forms the subject of this paper, was considered worthy of study on its own account. The work on the subject was discontinued when only approximate and partial results had been secured, but the publication of such data may at least call attention to some phenomena worthy of further study. Among the phases of the subject deserving further attention are the activity of the platinum metals other than those mentioned, a fuller comparison of the atuthor's results with those of Sackur, and the thickness and properties of the oxide fillus.

The author wishes to acknowledge his obligation to Mr. Joseph Morwitz for valuable assistance and to Dr. E. W. Tullotson for helpful suggestions.

${ }^{2}$ Industrial Fellow, Mellon Institute.

\$ Sitzb. Ges. Naturw. Marburg, 1907, p. 33.

On account of the brevity and inaccessibility of Wigand's report, the following translation is given:

"A letter from Wöhler (Göttingen) to Buff (Giessen) written in the year 1869 led me to conduct a series of experiments to explain the fact, also observed many other times, that the catalytic power of smooth platinum is considerably increased if it has been previously used as an electrode in a cell for the decomposition of water.

"In this preliminary paper information about the single experiments will be reserved until the quantitative relations are studied more exactly. The method of experimental procedure is as follows:

"In an eudiometer tube with electrodes of smooth platinum wire or foil, detonating gas is liberated from dilute sulfuric acid until the electrodes are completely surrounded by the gas. The connection with the battery is then broken. For several hours a gradual rise of the liquid is theo observed, as a rule until the platinum is completely covered.

"This phenomenon is explained in this way; as the result of the preceding galvanic polatization the platinum is loosened at the surface and heavily charged with hydrogen and oxygen. The power of catalyzing the combustion of detonating gas is increased in much the same way as if the platinum were platinized until black. After being well purified and heated to glowing, smooth platinum possesses this catalytic power only in a small degree."

So far as ascertainable, no further results have been published by Wigand. 
apparatus confirmed his results. The first object of the present investigation was to determine which electrode, if not both, acquires the increase in catalytic activity which Wigand found; next, it was desired to make the results quantitative, if possible, and the third aim was to determine the cause of the increased action. A number of secondary questions developed when the results of the experiments to be described were compared with those of Sackur." The most important of these secondary questions was that of the activity of other metals than platinum, and several brief experiments on those metals were included. A few experiments were also made on another class of reactions, namely, the hydrogenation of oils.

\section{Experimental.}

Platinum.-For the purpose of deciding which electrode acquires most of the increased activity shown by Wigand's research, an H-shaped electrolytic cell was made containing a stopcock in the transverse tube. The electrodes consisted of rolls of platinum gauze in contact with platinum wires sealed through the botton of the test-tubes. The cell was filled with $0.1 N^{\prime}$ sulfuric acid and was inverted over a vessel of the same solution. After passing a current of 0.1 ampere through the cell, keeping the stopcock open, until the tubes were nearly full of detonating gas, and allowing a minute for the diffusion of the gases, the stopcock was closed. It was inmediately noticed that the gases combined more rapidly in the tube containing the gauze which had served as anode.

With the expectation of making quantitative comparison, and insuring thorough mixing of the gases, several other forms of apparatus were so devised as to permit accurate measurement of the volume of gas. The difficulty of obtaining quantitative results, however, was quickly demonstrated. Wide variations in the time required for recombination of the gases in any form of apparatus were attributed to such factors as slight impurities on the surface of the liquid and variations in the amount of liquid adhering to the metal. Qualitatively, however, the same result was obtained in practically every case; the use of platinum as anode in an electrolytic cell (with dil. sulfuric acid) caused a marked increase, while its employment as cathode produced no decided change in its cata. lytic power.

The final form of apparatus, with which most of the experiments were made, consisted simply of an eudiometer tube of $50 \mathrm{cc}$. or $100 \mathrm{cc}$. capacity, and a piece of gauze or foil of the metal under examination, usually 2.5 cm. square. In the experiments on platinum, the metal was, with the exception to be noted later, of the quality used in laboratory ware. After giving the metal the treatment desired for the individual experiment, it was rolled into cyindrical form and inserted into the closed end of the

1. Z. Elektrochem, 12, 637 (1906); Z. physik. Chem., 54,641 (1906)。 
eudiometer tube. The tube was then filled with distilled water and inverted over water. The detonating gas, which had been generated in a larger vessel, was delivered into the eudiometer tube as needed. The time of activation of the metal varied considerably in the earlier experiments, but I 5 seconds was found to be sufficient to produce the maximum activation. As a rule, the electrolysis was continued considerably longer than ${ }_{5} 5$ seconds, especially when the metal was used as cathode. The current density was variable, and was not measured in most of the final experiments, but in general the activating current was about 0.3 ampere. In order to correct the volume of gas for temperature changes, if necessary, a blank tube containing air was placed alongside the others.

The following, one of the most extensive series of experiments, gave results which were characteristic of all those performed:

The general plan of this series was to compare the activity of catalyzers before and after "cathodizing," (a word coined for convenience to denote using the metal as cathode) in a number of successive experiments, then to compare results similarly before and after "anodizing," $i . e .$, using as anode. In each of 6 eudiometer tubes was put a piece of platinum gauze, $2.5 \mathrm{~cm}$. square, using the method already described. All pieces of gauze used in the preceding experiments were cleaned with chromate mixture, then heated to redness, but not treated electrically. In the first few experiments the total contraction will be given for each tube at a number of readings. Tubes Nos. I and 2 were of $100 \mathrm{cc}$. capacity, the rest $50 \mathrm{cc}$. The first readings, taken as soon as possible after filling the tubes, follow:

$0=9: 34$ A.M., Feb. 26, I9I4.

Total Contraction, $\mathrm{Cc}$.

$\begin{array}{crrrrrrr}\text { Minutes. Tube No. 1. } & 2 . & 3 . & 4 . & 5 . & 6 . & \text { Average. } \\ 15 & 0.50 & 2.10 & 1.90 & 3.70 & 15.92 & 5.19 & 4.9 \\ 30 & 6.45 & 3.70 & 3.36 & 7.05 & 27.25 & 11.03 & 9.8 \\ 45 & 8.91 & 5.25 & 4.70 & 10.48 & 36.20 & 17.23 & 13.8 \\ 60 & \text { II.45 } & 7.00 & 6.10 & 13.97 & 47.30 & 23.59 & 18.2\end{array}$

A second set of readings, taken a few hours later, gave the following results:

$0=1: 20$ P.M., Feb. 26.

$\begin{array}{crlllrrr}\text { Minutes. } & \text { Tube No. } 1 . & 2 . & 3 . & 4 . & 5 . & 6 . & \text { Average. } \\ 20 & 2.80 & 3.55 & I .80 & 2.56 & 6.70 & 4.05 & 3.5 \\ 40 & 4.90 & 6.32 & 3.30 & 5.45 & 14.07 & 7.97 & 7.0 \\ 60 & 6.80 & 8.70 & 4.95 & 8.16 & 23.95 & 11.90 & 10.7\end{array}$

The variations between the results with different pieces of gauze which were met with in all experiments, show the futility of trying to establish quantitative comparisons. Another set of readings at $4: 30$ P.M. gave an average rate of combination of $10.6 \mathrm{cc}$. per hour.

The next morning the following readings were taken: 
$0=9: 07$ A.M., Feb. 27.

Total Contraction, Co.

$\begin{array}{crrrrrrrr}\text { Minutes. } & 1 . & 2 . & 3 . & 4 . & 5 . & 6 . & \text { Average. } \\ 7.5 & 1.28 & 2.46 & 0.80 & 1.44 & 2.96 & 1.00 & 1.7 \\ x 5 & 2.32 & 4.80 & 1.50 & 2.69 & 5.95 & 1.82 & 3.2 \\ 22.5 & 3.90 & 6.17 & 2.15 & 4.44 & 9.20 & 2.61 & 4.7 \\ 30 & & 4.52 & 9.70 & 2.90 & 5.04 & 12.61 & 3.37 & 6.3 \\ \text { Average rate per hour.......... } & & 9.04 & 19.40 & 5.80 & 10.08 & 25.22 & 6.74 & 12.7\end{array}$

All gatuzes were then removed, used as cathode in the activating cell one minute with a current of 0.3 ampere, then replaced in their respective tubes. The next set of readings follows:

$0=$ I0:20 A.M., Feb. 27.

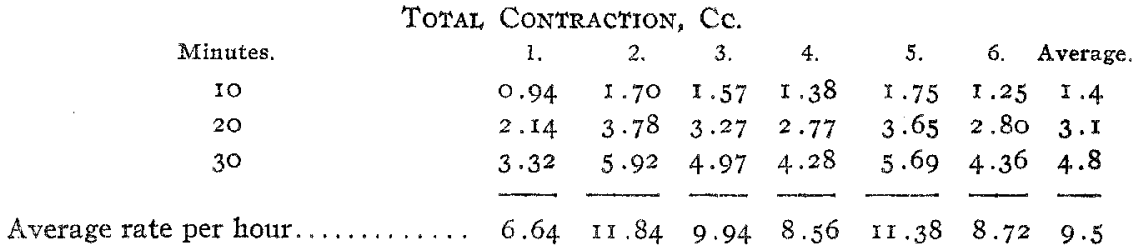

After the tubes had stood until March and and were refilled, the average rate was $6.35 \mathrm{cc}$. per hour. After one minute's cathodization the rate rose to $\mathrm{II} .4 \mathrm{cc}$. per hour. After a second treatment as cathode the rate was $9.25 \mathrm{cc}$. per hour. On March $3 \mathrm{rd}$, at 9 A.M., the rate was 8.5 cc. per hour before cathodizing and ro. $5 \mathrm{cc}$. per hour after cathodizing. Another cathodization at 4 P.M. of the same day changed the rate from 9.0 to $3.7 \mathrm{cc}$. per hour. The rate continued low during the next three days, and several cathodizations had the usual uncertain effect. In all other experiments, the result of cathodization was likewise variable.

Just before the first anodization in this series, the following readings were taken:

$0=\mathrm{I}: 23$ P.M., Mar. 6.

Total Contraction, $\mathrm{Cc}$.

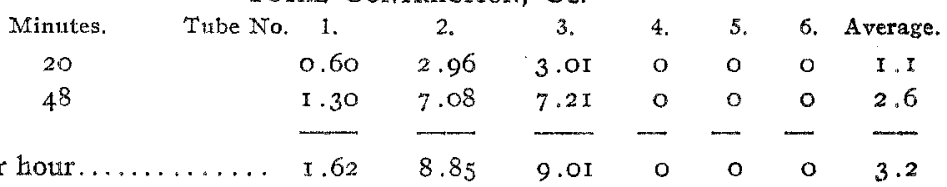

After giving each gauze one minute's anodization and refilling the tubes, the following readings were taken:

$0=3: 00$ P.M., Mar. 6.

\begin{tabular}{|c|c|c|c|c|c|c|c|}
\hline \multicolumn{8}{|c|}{ Totar Contraction, $\mathrm{Cc}$. } \\
\hline Minute.s. & Tube No. 1. & 2. & 3. & 4. & 5. & 6. & Average \\
\hline 5 & 6.58 & 2.88 & I. 78 & 4.00 & 4.65 & 2.50 & $3 \cdot 7$ \\
\hline 30 & 8.38 & 16.95 & 11.97 & 16.96 & 31.87 & 13.97 & 16.7 \\
\hline 40 & I0. 38 & 21.58 & I5.53 & 21.36 & 37.42 & 21.53 & $2 x .3$ \\
\hline 60 & $\times 4.86$ & 33.68 & 23.48 & 30.40 & ... & 33.60 & $27 \cdot 2$ \\
\hline
\end{tabular}


In this experiment, the rate for the first hour was increased 8.5 times by anodization. Further readings showed a steady decrease in activity. At 9 A.M. March 7 , when the rate had dropped to $6.8 \mathrm{cc}$. per hour, 5 minutes' anodization accelerated the average rate to $24.3 \mathrm{cc}$. per hour. The next day the result was substantially repeated. By March ro, however, further anodization no longer increased the rate, apparently on account of a film of grease which had reached the gauze.

In other series of experiments the order of the kinds of treatment was varied. There were often marked deviations between the results of individual experiments, but the bulk of observations led qualitatively to the same conclusion, that the use of the metal as anode very materially increases its subsequent effect on the rate of combination of detonating gas, while the effect of use as cathode is uncertain.

From a few experiments the further conclusion was tentatively established that the activity acquired on oxidation was lost on reduction, more slowly that it was acquired. A single experiment in which an alternating current was used in the activating cell gave the anticipated result, namely, a net increase in activity.

In a few experiments the usual piece of gauze was platinized, then treated similarly to the smooth platinum. The rate was always much greater than with smooth platinum. Use as cathode again gave uncertain results, but use as anode increased the rate of combination very materially, producing an explosion in one experiment.

It has already been pointed out that the platinum used was of commercial quality. Some experiments with a piece of c. P. platinum foil of the same size also showed increased activity after anodization.

Other experiments in which fresh pieces of platinum foil were used as catalyzers, before and after direct treatment with nitric acid instead of electrolysis, showed that the increase in activity is due simply to oxidation of the surface and is not peculiarly the effect of electrolysis.

Palladium.-This metal was selected next, on account of its great activity as a catalyst and its high adsorptive power for hydrogen. Some pieces of palladium foil of the same size as those of platinum were treated and used according to the methods already described. The rate of combination, always much greater than with smooth platinum under comparable conditions, was always increased by anodization, while the effect of cathodization was small and variable. When the metal was freshly oxidized, an explosion usually resulted within 2 minutes after filling the tube, while an explosion never occurred when the palladium had been heated immediately before the experiment, and rarely when it had been used as cathode or immersed in gaseotus hydrogen.

Iridium.-The only specimen of iridium available at the time of the experiments was in the form of small lumps. An attempt was made to 
use these in the apparatus by attaching them to other materials. No catalytic activity was observed, but this result should not be regarded as conclusive.

Gold.-Gold foil, C. P., when used as catalyzer in the same apparatus, was found to have a catalytic power very much less than that of platinum but still noticeable. In one experiment with untreated gold $0.5 \mathrm{cc}$. of gas in a $50 \mathrm{cc}$. tube combined in $2 \mathrm{I}$ days. The effect of oxidation was very pronounced; in contact with the same piece of gold, anodized, almost the entire $50 \mathrm{cc}$. combined in 7 days. Anodization of the gold imparted a reddish color to the surface, followed by a change to greenish black when the metal was put into the reaction mixture. The surface, then, was greatly increased.

Cobalt.-Cobalt was the only other metal which was found to possess unmistakable catalytic activity for the reaction. The specimen of this metal was in the form of one cm. cubes and was not of known purity. Untreated, this metal combined $25 \mathrm{cc}$. of detonating gas in 90 days; anodized, it combined $50 \mathrm{cc}$. of gas in between 40 and 70 days. On anodization the solution immediately began to acquire the pink color of cobalt ion, and the surface of the metal became rougher.

Other Metals.-Experiments were also made with silver, copper, leac, th, nickel, iron, chromium, tungsten, manganese, aluminum and bismuth. Two pieces of each metal were used, one untreated and one anodized. The first 4 mentioned were in the form of foil; the tin, aluminum and nickel were in sheet form, and the rest were in irregular pieces. All but the iron were of "C. P." grade. Each specimen of metal was inserted in an eudiometer tube and left in contact with the gas mixture for 3 months. To eliminate temperature effects, diffusion, catalysis by the glass wall, etc., two additional tubes without metal were filled, one with detonating gas and the other with air, and corresponding corrections were made. In some of the tubes a contraction was observed, amounting in the end to nearly $1 / 3$ of the total volune, and the residue proved to be largely bydrogen. Therefore, they were simply cases of oxidation of the metal. The manganese liberated some hydrogen from the water. There was no evidence of catalytic action by any of the metals in this series of experiments.

Eydrogenation of Oils.-The hydrogenation of certain vegetable and animal oils was chosen as another example of catalysis in which results might be of interest. The customary catalyst for such reactions is a finely divided metal or oxide, but it was expected that although the amount of hydrogenation with sheet metal as a catalyzer would not be great, sheet palladium would easily give measurable results.

Two series of experiments on the hydrogenation of cottonseed oil were conducted. The temperature in each experiment was $85^{\circ}$ to $90^{\circ}$, the 
pressure $50 \mathrm{lbs}$, the time one hour, and the weight of oil $15 \mathrm{~g}$., with a piece of smooth palladium $2.5 \mathrm{~cm}$. square as catalyzer. In the first experiment in each series the palladium was heated before using; before the second it was cathodized until saturated with hydrogen; before the third it was anodized Io or 15 minutes. The oil to be hydrogenated was contained in a small flask which was rotated horizontally within the pressure cylinder. The iodine number of the product was determined according to Wijs' method. In the following table of iodine numbers found, each figure represents the mean of two results which agreed within a few tenths of a unit:

\begin{tabular}{|c|c|c|}
\hline Original sample. & $\begin{array}{c}\text { Ist Series. } \\
\text { I06. }\end{array}$ & $\begin{array}{c}\text { 2nd Series. } \\
\text { 106. }\end{array}$ \\
\hline 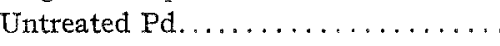 & IOI. 6 & 104.0 \\
\hline Cathodized Pd................ & xor. 4 & IOI 4 \\
\hline Anodized $\mathrm{Pd}, \ldots \ldots \ldots \ldots \ldots \ldots \ldots$ & 84.5 & 87.0 \\
\hline
\end{tabular}

The experiments were then repeated, with palladized palladium as catalyzer. The hydrogenation did not proceed as far as with the smooth metal, and the effect of oxidation, although noticeable, was much smaller. The product of hydrogenation, under the same conditions as in the preceding experiments, but using untreated palladized palladium as catalyzer, had an iodine number of 105; when the catalyzer had been anodized, the iodin number of the product was 103.4.

Similar experiments were made on castor oil, fish oil, seal oil and linseed oil, using smooth palladium and appropriate temperatures and pressures for each, but the hydrogenation found was always very slight. No effect on cottonseed oil was observed when sheet nickel was used as catalyst.

\section{Discussion.}

Wigand's theory that the increased activity is due simply to a loosening of the surface is disproved by the fact that no surface change was noticeable on anodizing, while in some of the earlier experiments a darkening of the surface was obtained by cathodizing with higher current densities than that usually employed, without very greatly increasing the activity.

The most reasonable possibility is, then, that the activity may be due to oxidation of the platinum. Several anthors have noted that a small amount of platinum is dissolved or rendered soluble by oxidizing agents. Marie found that if platinum is treated with strong oxidizing agents or used as anode in an electrolytic cell, then washed and treated with acids containing reducing agents, $e . g$. , hydrochloric acid containing a small amount of potassium iodide, a small but measurable quantity of platinum

${ }^{1}$ Compt. rend., I45, II7 (1907); I46, 475 (1908); J. chim. phys., 6, 596 (1908). Complete references to earlier work by other investigators are given in the last mentioned paper. 
is dissolved. In making quantitative measurements, the platinum was determined colorimetrically. Using pieces of platinum of various grades, $2.5 \mathrm{sq} . \mathrm{cm}$. in area, as anode in dil. sulfuric acid and other electrolytes, for 30 to $\times 20$ hours, at a current density of approximately 4 amperes per square decimeter, Marie obtained 0.0005 to $0.0010 \mathrm{~g}$. of platinum subsequently dissolved by the hydrochloric acid and potassium iodide, and found increases in weight of the anode amounting to $0.000 \mathrm{r}$ to $0.0003 \mathrm{~g}$. during the process of electrolysis. When a piece of platinum of the same total area was treated with any one of a number of oxidizing agents for a number of hours, the amount of platinum subsequently dissolved was about $0.00004 \mathrm{~g}$. In both cases the extent of oxidation had apparently reached a maximum, due to the protective action of the film of oxide, which he believes to be of the formula $\mathrm{PtO}_{2}$. In some of the experiments a slight brownish color was noticed.

The author calculated the thickness of the film of oxide or rather of the platinum which had become soluble, using Marie's figures, on the assumptions that the surface is smooth and the thickness of the film is uniform. From the above figures and the density of platinum, 2I.5, we obtain in the case of electrolysis:

$$
\frac{0.0007 \text { (av.) }}{2 \mathrm{I} .5 \times 25}=\mathrm{I} .3 \times \mathrm{ro}^{-6} \mathrm{~cm} \text {., }
$$

and in the case of treatment with oxidizing agents:

$$
\frac{0.00004}{21.5 \times 25}=7 \times 10^{-8} \mathrm{~cm} \text {. }
$$

The atomic diameter in solids according to a number of investigators is : to $3 \times 10^{-8} \mathrm{~cm}$. Therefore, the soluble film formed by treatment with oxidizing agents has the thickness of two or three atoms, or possibly of only one atom, as the surface may not be entirely smooth and may be actually greater than that found by direct measurement. The conditions at the surface of the metal, if the layer is only one atom thick, are identical with those observed and discussed by Langmuir. ${ }^{1}$ The thickness of the film after prolonged electrolytic treatment, however, amounts to that of 30 or 40 layers of atoms, so in that case there may be some loosening of the surface before a fully protective film is formed. The author did not determine the thickness of the film after the brief electrolytic treatment in his experiments.

According to Marie, surface oxidation of platinum by chemical or electrolytic means gives to the surface layer some of the properties of platinic oxide which he enumerates. To his list is to be added the property of catalytic power greater than that of metallic platinum, easily evident even when the film is of little more than atomic thickness.

1 THIS JOURNAL, 38,2267 (x916). 
This conclusion is not in agreement with that of Sackur, ${ }^{1}$ who deduced from a number of experiments on residual currents and on the amount of gases given off on electrolysis when different metals as electrodes, that the catalytic power of metals is decreased by negative polarization and increased by positive polarization. According to his view, oxygen acts on platinum as a poison, and the more easily a metal becomes passive, the lower is its catalytic power. The conditions in the author's experiments differ in many aspects from those in Sackur's; it must be noted, however, that the reactions in both cases take place in aqueous solution; in the one case in a thin film, and in the other in a large volume. On consideration of Sackur's deductions, without repeating his experiments, no tangible ground for adverse criticism of most of his conclusions was presented, but the mass of direct evidence in the author's experiments points to the opposite conclusions.

According to Sackur, the order in which the metals studied stand with respect to their catalytic activity toward the combination of hydrogen and oxygen, beginning with the most active is: silver, platinum, copper, lead, tin, nickel, chromium. Gold and the other platinum metals were not included in his experiments. According to the author's results, the order is palladium, platinum, cobalt, gold, with no indication of activity by any other metal studied. The activity of palladium is increased by surface oxidation for apparently the same reasons as is that of platinum. The activity of gold is increased in a higher ratio, on account of the formation of a non-protective coating of oxide, which is later reduced to the finely divided metal. The increased activity of cobalt is apparently due simply to etching and increase of suriace. An estimation of the comparative order of magnitudes of the activities of the metals used gives 50 to $x$ for the ratio of palladium to platinum; 2000 to $\mathrm{I}$ for platinum to gold, and 200 to $\mathrm{I}$ for platinum to cobalt.

\section{Summary.}

$x$. The catalytic activity of platinum and of palladium toward the reaction between hydrogen and oxygen at ordinary temperature in the presence of a film of water is increased by surface oxidation of the metal, whether accomplished by anodic polarization or by chemical means.

2. This surface oxidation consists in the formation of a film of surfacecombined oxygen of little more than atomic thickness, whereby the surface acquires some of the properties of an oxide of the metal.

3. The catalytic activity of smooth palladium toward the hydrogenation of cottonseed oil is increased by surface oxidation.

4. The effect of cathodic polarization or hydrogenation on the catalytic activity of platinum and palladium is slight and uncertain in direction.

${ }^{1}$ Loc. cit. 
5. The order of activity among the metals tested, beginning with the highest, is: palladium, platinum, cobalt, gold, with large differences in the order of magnitude of the activity. The activity of a number of other metals is too small to be discernible. The activity of gold is increased by surface oxidation, largely on account of increase of surface.

6. The results obtained are at variance with Sackur's conclusions with regard to the passivity of these metals and of hydrogen.

Pintaburar, Pa.

[Contribution from the DePartment of Analytical Chemistro, SheFfield ScIENTIFIC SchOOL, YaLE UNIVERSITY.]

\section{ANALYTICAL WEIGHING.}

BY HORACE I. WELLS.

Received January 3, 1920.

\section{Introduction.}

It appears that many chemists at the present time regard the use of long swings of the analytical balance as the best and most accurate method of weighing. Many recent text-books on quantitative analysis advocate this process, sometimes without even mentioning a simpler method, and the teaching of long swing weighing has evidently grown in favor in recent times.

While admitting that the use of long swings of the balance is capable of giving results that are accurate enough for ordinary purposes, the writer has absolutely no doubt that this practice is generally somewhat less accurate than the ordinary, simple method of using short swings. The very serious objection to the method of long swings, however, is that it is more difficult and much slower than the common method.

The object of this article, therefore, is to discuss methods of weighing, to present a plea for the general use in teaching and in practice of the simple, easy and accurate process of weighing with short oscillations of the balance, and for the abandonment of long swing practice, which evidently involves a great waste of time and labor, with no gain, but probably some loss, in accuracy.

The advocates of long swing weighing usually recommend the finding of a "zero-point" of the empty balance--a point not coincident with the zero of the graduated scale-in connection with each weighing, thus largely increasing the burden of labor in their otherwise cumbersome process. It is believed that this practice should be given up, no matter what method of weighing is employed, in favor of using the apparent zero of the balance as the basis of weighing, according to the old and reliable custom; for it is certain that when we weigh by difference, as we almost invariably do, the point of equilibrium of the empty balance is of no consequence, provided 